MODELING, IDENTIFICATION AND CONTROL, 1989, VOL. 10, NO. 1, 3-11

doi:10.4173/mic.1989.1.1

\title{
Frequency response adaptive control of a refrigeration cycle
}

\author{
JENS G. BALCHEN, KÅRE TELNES and DAVID DI RUSCIO†
}

Keywords: Adaptive control, self tuning, frequency response, refrigeration cycle.

\begin{abstract}
A technique for the adaptation of controller parameters in a single control loop based upon the estimation of frequency response parameters has been presented in an earlier paper. This paper contains an extension and a generalization of the first method and results in a more versatile solution which is applicable to a wider range of process characteristics. The application of this adaptive control technique is illustrated by a laboratory refrigeration cycle in which the evaporator pressure controls the speed of the compressor.
\end{abstract}

\section{Introduction}

Many types of schemes and algorithms for adaptive control have been proposed and demonstrated in practical use. One such technique is based upon online identification of the most essential frequency response parameters of a single feedback control loop. This has been presented in a paper by Balchen and Lie (1986). The basic principles of this technique will be reviewed below. Its main advantages are that:

It requires very few mathematical/numerical operations, i.e. only a short computer program is required for the algorithm.

The sinusoidal perturbation of the system adjusted according to the natural system disturbance level, guarantees proper excitation for identifiability.

The system is robust and has a satisfactory convergence speed.

The system is easy for process operators to comprehend and adjust.

The following shows how the adaptive control scheme is applied to the control of a refrigeration cycle. This is a very common industrial process, particularly in the petroleum-, petrochemical- and foodprocessing industries.

\section{Basic principles of adaptive control}

The control loop is assumed to consist of a process with a scalar frequency response $h_{p}(j \omega)$ and a controller with frequency response $h_{c}(j \omega)$. The loop frequency response is $h_{0}(j \omega)=h_{p}(j \omega) h_{c}(j \omega)$. In Fig. 1 three important frequencies are indicated in a polar plot of $h_{0}(j \omega)$, namely the 'cross-over frequency' $\omega_{c}$, the $\omega_{180}$ yielding a phase shift of $-180^{\circ}$, and a frequency denoted $\omega_{x}$ at which the 'disturbance

Received 12 December 1988.

† Division of Engineering Cybernetics, The Norwegian Institute of Technology, N-4034 Trondheim, Norway.

This article was presented at the IFAC International Symposium on Adaptive Control of Chemical Systems (ADCHEM) held at Lyngby, Copenhagen, Denmark, August 17-19, 1988 and is reprinted with the permission of IFAC. 


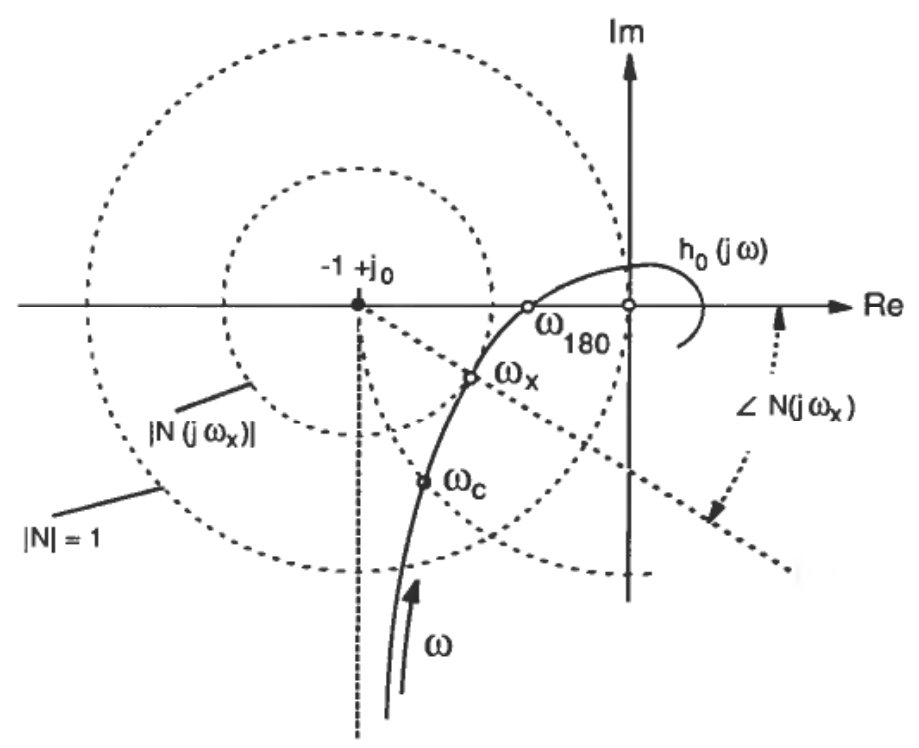

Figure 1. The loop frequency response $h_{0}(j \omega)$ and associated characteristic parameters.

reduction ratio'

$$
N(j \omega)=\frac{1}{1+h_{0}(j \omega)}
$$

has a certain prescribed value. The adaptive control scheme proposed here, is such that it experimentally determines frequency $\omega_{x}$ and adjusts the parameters of a PIDcontroller so as to arrive at the prescribed values of $\left|N\left(j \omega_{x}\right)\right|$ and $L N\left(j \omega_{x}\right)$. In the original version of the algorithm, presented in Balchen and Lie (1986), the frequency $\omega_{x}$ was chosen to be $\omega_{180}$, that is $\angle N\left(j \omega_{180}\right)=0$ was prescribed. This choice is perfectly acceptable in case of processes with a dominant transportation lag or nonminimum phase behaviour. However, in processes with a less negative phase shift in the region of the crossover frequency, it is more desirable to be able to specify $\angle N\left(j \omega_{x}\right)$ to some non-zero value, for example 20-60 degrees. In the adaptive control scheme, the proportional gain of the controller $\left(K_{p}\right)$ is adjusted so as to arrive at a prescribed value of $\left|N\left(j \omega_{x}\right)\right|$ for example in the range 1-2. The integral time, $\left(T_{i}\right)$ and the derivative time $\left(T_{d}\right)$ of the controller are adjusted to fixed ratios of $1 / \omega_{x}$. This simple algorithm will be adequate for most industrial applications.

The adaptive algorithm contains the following functions:

Estimation of $\omega_{\mathbf{x}}$.

Estimation of $\left|N\left(j \omega_{x}\right)\right|$.

Estimation of the variance of the control error caused by process disturbances.

Adjustment of controller proportional gain.

Adjustment of controller integral-and derivative-times.

Adjustment of sinusoidal perturbation signal amplitude.

A block-diagram of the whole adaptive control system is shown in Fig. 2. The different functions listed above are identified with capital letters $\mathbf{A}-\mathbf{F}$, referring to the list 


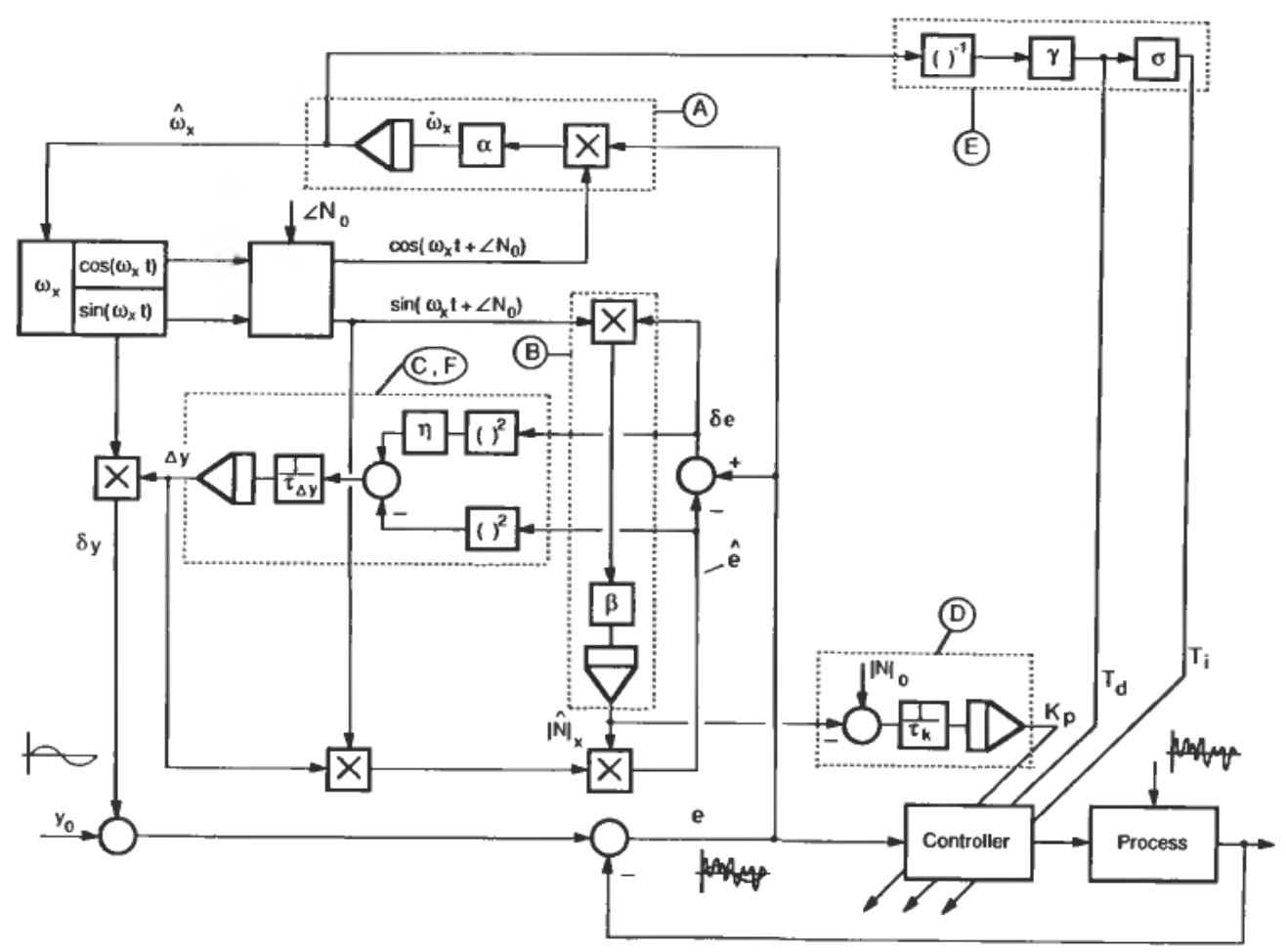

Figure 2. Block diagram of the frequency response of the adaptive controller.

above. The functions are explained as follows (see Balchen and Lie (1986) for further details):

A. The proper frequency $\hat{\omega}_{x}$ is found when the cross-correlation between the sinusoidal component of the control deviation $(e)$ and the sinusoidal reference signal $\cos \left(\hat{\omega}_{x} t+\angle N_{0}\right)$ is equal to zero. A simple gradient loop with gain $\alpha$ adjusts $\hat{\omega}$.

B. The proper estimate $|\hat{N}|$ is found when there is no cross-correlation between the residual $\delta e=e-\hat{e}$ and the sinusoidal perturbation signal $\sin \left(\hat{\omega}_{x} t+\angle N_{0}\right)$. A simple gradient loop adjusts $|\hat{N}|$.

D. The adjustment of the controller proportional gain $\left(K_{p}\right)$ is achieved by a simple integral controller with gain $1 / \tau_{k}$ and with setpoint $|N|_{0}$.

E. The time dependent parameters of the controller are directly adjusted as follows:

$$
T_{d}=\gamma \frac{1}{\hat{\omega}_{x}}, \quad T_{i}=\sigma T_{d}
$$

C, F. It is advantageous to adjust the perturbation signal $(\Delta y)$ so that the mean square value of the sinusoidal component $(\hat{e})$ is a certain fraction $(\eta)$ of the total control deviation $(e)$. The adjustment of the perturbation amplitude is achieved by a simple gradient loop driving the quantity $\eta(\delta e)^{2}-(\hat{e})^{2}$ towards zero.

The parameters $\alpha$ and $\beta$ in the estimator, determine the rate of convergence of the two estimation loops for $\hat{\omega}_{x}$ and $|\hat{N}|$. Because the speed of convergence is closely related to the bandwidth of the control system, which in turn is closely related to $\hat{\omega}_{x}$, both these parameters could either be given constant values by making $a$ priori guesses of $\hat{\omega}_{x}$, or be updated by a simple updating scheme. The same argument 


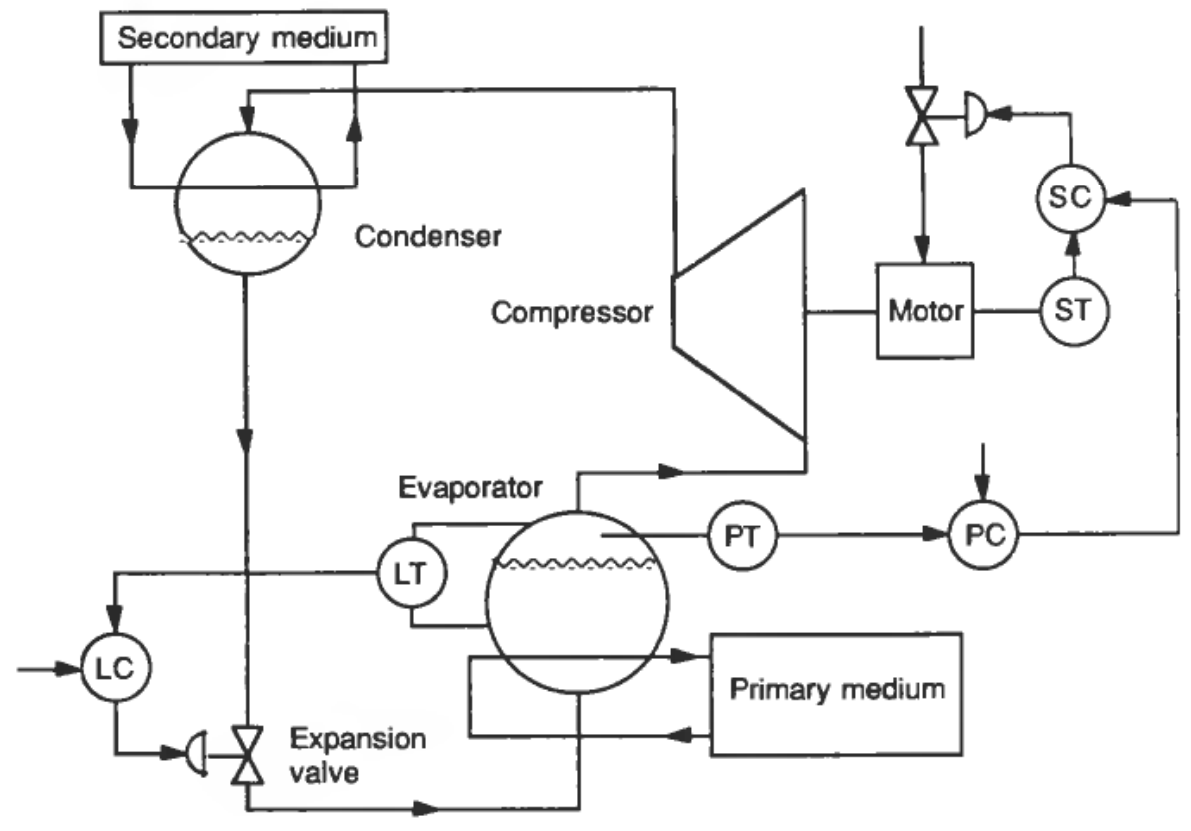

Figure 3. Refrigeration cycle with two control systems.

applies to the two parameters $\tau_{k}$ and $\tau_{\Delta y}$ associated with the speed of convergence of factors $K_{p}$ and $\Delta y$ respectively. Thus it is possible to design this adaptive controller so that the operator does not have to do any difficult initial parameter evaluation except determine the order of magnitude of the process time constants.

\section{Control of refrigeration cycles}

A simple refrigeration cycle is shown in Fig. 3. It consists of four major units: A compressor driven by a motor with speed control.

An evaporator in which refrigerant boils under low pressure, taking heat energy away from the primary medium to be cooled.

A condenser in which the compressed refrigerant vapour is condensed into liquid by the cooling effect of the secondary medium which is being heated.

An expansion valve allowing the high pressure liquid refrigerant in the condenser to expand into the low pressure evaporator in an adiabatic manner thereby closing the cycle.

There are two main control variables in this cycle:

The opening of the expansion valve.

The set-point of the compressor speed control system.

In addition, control valves can be installed both in the primary and the secondary medium circuits.

A number of control structures can be applied to the cycle in Fig. 3 depending on the use of the system. The two most important applications of the refrigeration cycle are: 
Frequency response adaptive control
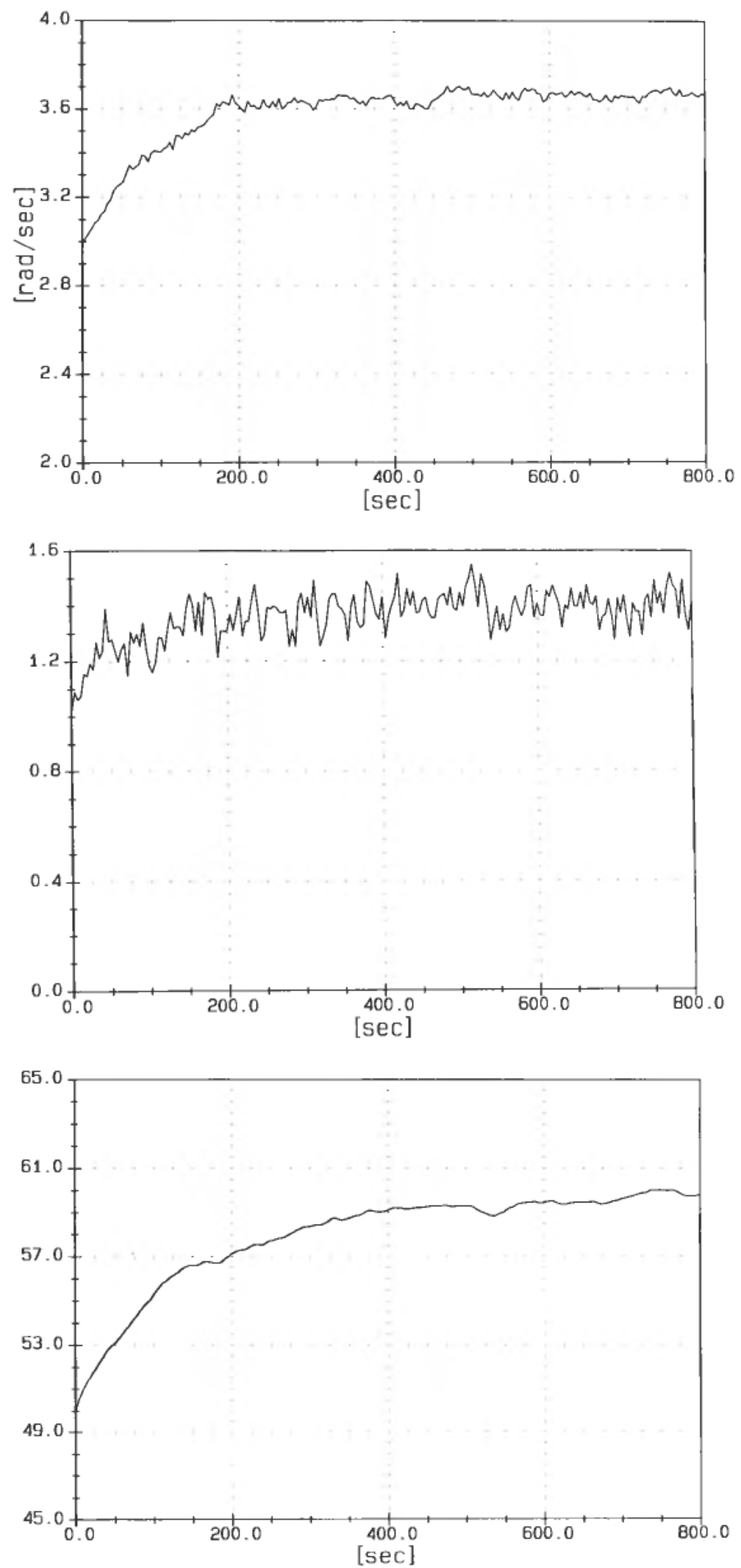

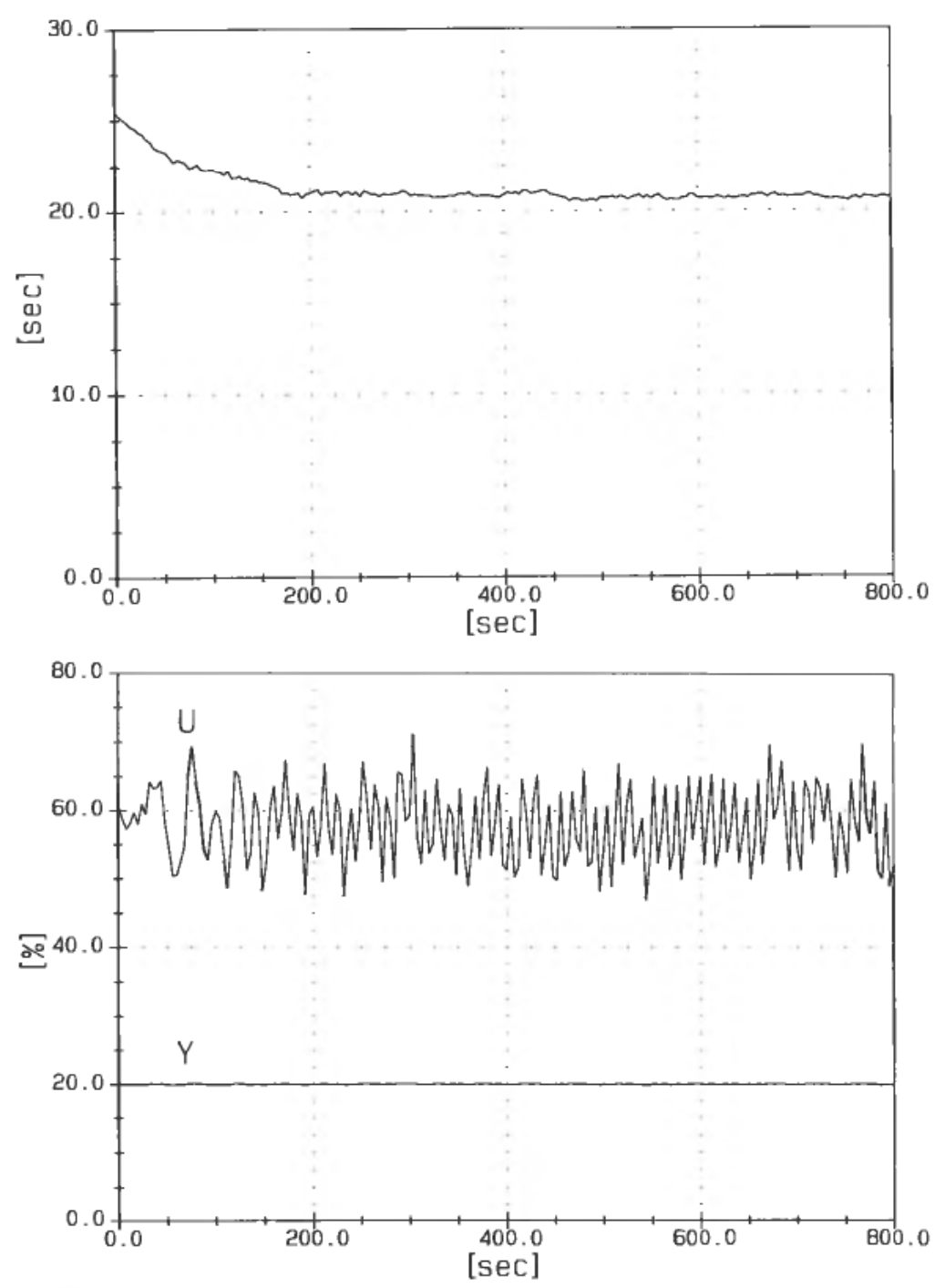

Figure 4. Responses of the adaptive control system after it is turned on at time equal to 0 . (a) estimated $\omega(t),(b)$ estimated $N_{x}(j \omega),(c)$ adapted $K_{p},(d)$ adapted $T_{i}$ and $(e)$ measurement $Y$, and controller output.

A cooling system for the primary medium.

A heat pump to bring low temperature energy from the primary medium to more valuable high temperature energy in the secondary medium by adding energy to the compressor.

In small home refrigerators the most common solution is to control the expansion valve from the pressure in the evaporator, thereby controlling the evaporator temperature. At the same time the compressor is run at a constant speed in an on-off mode controlled by the temperature in the primary medium. In larger, industrial systems where the capacity of the compressor can be continuously adjusted by means of speed control (as in Fig. 3), a structure like that indicated in Fig. 3 is common. Here the level of the refrigerant in the evaporator is maintained by means of a level control system acting upon the expansion valve. Furthermore, the pressure 
Frequency response adaptive control
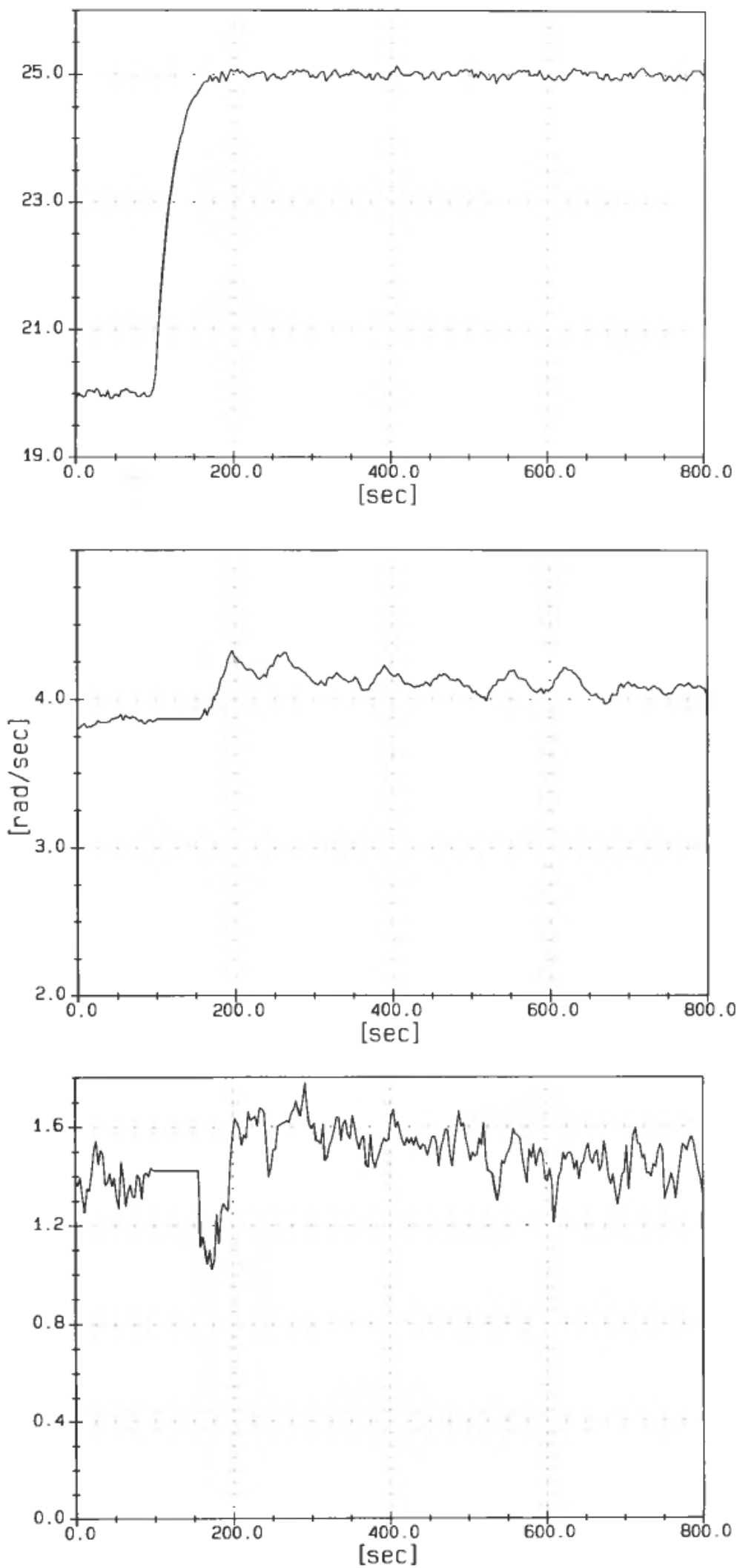

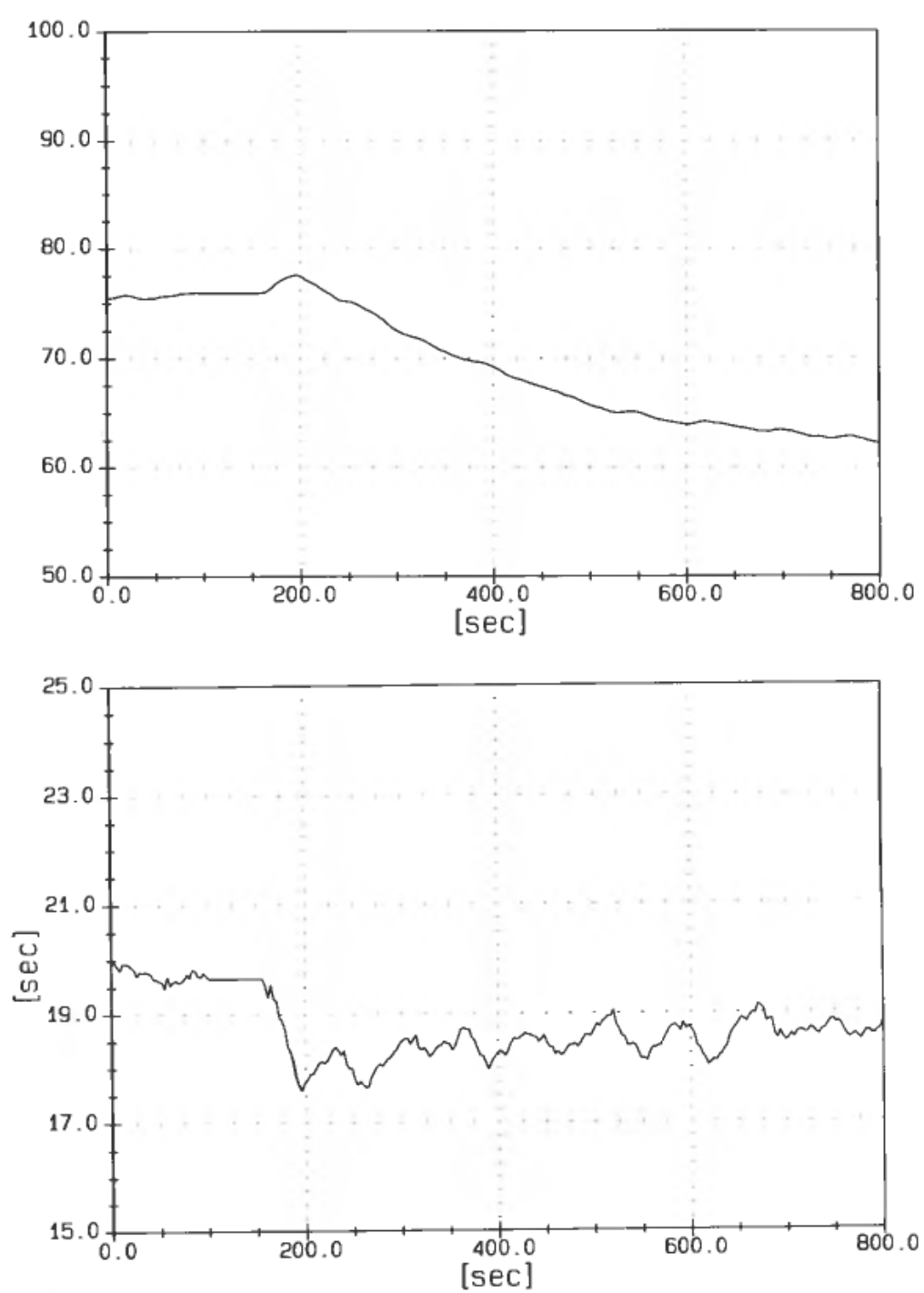

Figure 5. Responses of the adaptive control system after a step change in the evaporator pressure setpoint from 20 to 25 per cent is applied at time equal to $100 \mathrm{~s}$. (a) measurement, $Y,(b)$ estimated $\omega_{x}(t),(c)$ estimated $N_{x}(j \omega),(d)$ adapted $K_{p}$ and $(e)$ adapted $T_{i}$.

in the evaporator (and thereby the temperature) is maintained by a pressure control system acting upon the setpoint of the compressor speed. The pressure in the condenser (and thereby the temperature) is floating and will adjust itself according to the temperature in the secondary medium. The evaporator acts like a boiler which has the so called 'shrink and swell effect'. This means that the amount of vapour bubbles in the liquid influences the liquid level in the evaporator. To secure good heat transfer between the tubes carrying the primary medium and the refrigerant in the evaporator, it is important that the level of refrigerant is always above the tubes. As a result, the level control is applied through the expansion valve.

This paper investigates the control of the pressure, and thereby the temperature, in the evaporator unit by the manipulation of compressor speed. This particular control loop is characterized by a dominant first order behaviour resulting in good 
robust control even without adaptivity in the controller. However, since the phase shift in the process only goes beyond $-90^{\circ}$ at fairly high frequencies, this process serves as a good example of why it is necessary to specify $\angle N\left(j \omega_{x}\right)=20-60^{\circ}$. In such cases, it makes sense to specify a rather low value of $\left|N\left(j \omega_{x}\right)\right|$ for example $0.7-1.5$ in order to avoid too high controller gain. High controller gain will inevitably lead to large and rapid control actions (compressor speed variations) which do not produce anything.

\section{Experimental test of adaptive controller}

The experimental test was performed on a laboratory refrigeration cycle with a compressor rated $5 \mathrm{~kW}$, a heating capacity of the primary medium about $15 \mathrm{~kW}$ and a cooling capacity of the secondary medium of about $20 \mathrm{~kW}$. The refrigeration cycle can be controlled by either conventional electronic analogue controllers or by computer control through an IBM PC/AT. The control program is written in language $\mathrm{C}$ using MS-DOS and the sampling period is $0.1 \mathrm{~s}$. Figure 4 illustrates a number of responses of the total system when adaptive control is turned on at a time equal to 0 . Figure 5 shows the responses of the adaptive system when the pressure setpoint is given a step change from 20 to 25 per cent at a time equal to $100 \mathrm{~s}$.

The parameters used in the experiments: were $\Delta y=0.08, \alpha=1 \cdot 0, \beta=5.0$, $\mu \sigma=76, \tau_{\Delta y}=10, \tau_{k}=4 \cdot 0,|N|_{0}=1 \cdot 4, \angle N_{0}=10$ and $y_{0}=20$ per cent.

\section{Conclusions}

The algorithm for modified frequency response adaptive control was tested and found as satisfactory as expected. The estimation of $\omega_{x}$ at any specified angle $\angle N\left(j \omega_{x}\right)$ is rapid and robust and constitutes the basis of the method. The estimation of $\left|N\left(j \omega_{x}\right)\right|$ may be somewhat dependent upon the shape of the loop frequency response $h_{0}(j \omega)$, particularly if the function contains a resonance phenomenon as in the refrigeration cycle under investigation. In such cases the response speed of the $|N|$-estimation loop should be somewhat reduced.

The present method is well suited to both sequential and simultaneous estimation and the adaptation of many control loops in a complex plant. In some cases it may only be desirable to monitor the frequency response properties of each control loop as given by the estimation algorithm without actually adapting the controller parameters. This may be a natural step before implementing full adaptivity.

\section{ACKNOWLEDGMENT}

Part of these investigations has been supported by the Royal Norwegian Council for Scientific and Industrial Research through project IT-3.32.18009.

\section{REFERENCE}

BALCHEN, J. G. and LiE, B. (1986). An adaptive controller based upon on continuous estimation of the closed loop frequency response. IFAC Workshop on Adaptive Systems in Control and Signal Processing, Lund, Sweden, July 1986, Pergamon Press 1987. Also in Modeling, Identification and Control, (1987) 8, 223-240. 